\title{
A presença do fisioterapeuta na puericultura no olhar dos profissionais de uma unidade de saúde da família
}

\author{
Olivia Galvão Lucena Ferreira1, Turrania Talita Soares de Castro², Simone Felipe Santiago², \\ Sandra Fernandes Pereira Meló ${ }^{3}$, Egmar Longo Araújo de Melo ${ }^{1}$, Verbena Santos Araújo ${ }^{4}$
}

\begin{abstract}
RESUMO
Este estudo objetivou conhecer a importância da atuação do fisioterapeuta na puericultura, na visão dos profissionais de saúde de uma unidade de saúde da família. Trata-se de um estudo descritivo, exploratório, com abordagem qualitativa. Foram entrevistados 25 profissionais de uma Unidade de Saúde da Família em João Pessoa, Paraíba. Todos entrevistados reconheceram a importância do fisioterapeuta na puericultura e a análise de conteúdo a respeito da visão destes sobre a atuação do fisioterapeuta na puericultura permitiu a organização de quatro categorias: Diagnóstico e tratamento precoce de crianças; Prevenção de doenças e outros agravos; Orientações às mães; e Avaliação do desenvolvimento das crianças. Apesar de não existir na unidade estudada o fisioterapeuta atuando com a equipe multidisciplinar, os profissionais desta unidade percebem que a inserção do fisioterapeuta junto à equipe atuando na puericultura seria de grande valor, principalmente para a integralidade do cuidado a saúde das crianças.
\end{abstract}

Descritores: Cuidado da Criança; Cuidado do Lactente; Fisioterapia; Atenção Primária à Saúde.

\section{How professionals in a family health unit view the partipation of physical therapists in childcare}

\begin{abstract}
This work aimed to analyze the importance of the physiotherapist in Well-child care within a unity family healthcare according to the vision of the health professionals. A descriptive study with exploratory and qualitative approaches was conducted. For that, a survey was performed with twenty-five professionals from a unity family health care in João Pessoa city, Paraíba, Brazil. All of the respondents recognized the importance of the physiotherapist in Well-child care, and the analysis about the vision of the public on the role of the physiotherapist in that field allowed the organization of four categories: Early diagnosis and treatment of children; Prevention of diseases and other health problems; Orientation to mothers; and Evaluation of child development. Although the unity family healthcare chosen for this study does not present a physiotherapist within the multidisciplinary team, the professionals have realized that, the insertion of the physiotherapist for acting in well-childcare in partnership with the group would be of a great value, especially for the integrality of the children's healthcare.
\end{abstract}

Descriptors: Child Healthcare; Infant Healthcare; Physiotherapy; Primary Healthcare.

\footnotetext{
${ }^{1}$ Fisioterapeuta pela Universidade Federal da Paraíba (UFPB), João Pessoa, PB, Brasil.

${ }^{2}$ Fisioterapeuta pela Faculdade de Ciências Médicas da Paraíba (FCM-PB), João Pessoa, PB, Brasil.

${ }^{3}$ Fisioterapeuta pelo Institutos Paraibanos de Educação (IPE), João Pessoa, PB, Brasil.

${ }^{4}$ Especialista em Gestão em Saúde pela Universidade Estadual da Paraíba (UEPB), Campina Grande, PB, Brasil.
} 


\section{Introdução}

O Sistema Único de Saúde (SUS) garante o direito à saúde com acesso integral, universal e gratuito para toda a população do país ${ }^{1}$. As crianças devem ter acesso a cuidados básicos de saúde para mantê-las saudáveis e capazes de alcançar todo seu potencial. Através do Estatuto da Criança e do Adolescente $(E C A)^{2}$, a legislação brasileira reforça o compromisso da promoção e do bem-estar dos mesmos onde esta responsabilidade não é apenas da família, mas do Estado e da sociedade como um todo. Garante ainda, a toda criança e adolescente, o direito a proteção à vida e à saúde, mediante a efetivação de políticas sociais públicas que permitam o nascimento e o desenvolvimento sadio e harmonioso, tendo condições dignas de existência, sendo que os cuidados com a saúde infantil estão entre as ações essenciais, previstas no ECA.

O conjunto de técnicas empregadas para assegurar o perfeito desenvolvimento físico e mental da criança, desde o período de gestação até a idade de 4 ou 5 anos e, por extensão, da gestação à puberdade é definido como puericultura. Seus objetivos básicos contemplam a promoção da saúde infantil, prevenção de doenças e educação da criança e de seus familiares, por meio de orientações antecipatórias aos riscos de agravos à saúde, podendo oferecer medidas preventivas mais eficazes. As ações compreendem ainda o acompanhamento periódico e sistemático das crianças para avaliação de seu crescimento e desenvolvimento, vacinação, orientações às mães sobre a prevenção de acidentes, aleitamento materno, higiene individual e ambiental e, também, identificação precoce dos agravos, com a finalidade de uma intervenção efetiva e apropriada ${ }^{3,4,5}$.

No Brasil, a puericultura está entre as ações programáticas mais ofertadas por serviços básicos de saúde, principalmente na Estratégia de Saúde da Família (ESF) ${ }^{6}$. Para a integralidade do cuidado e assistência à saúde das crianças na atenção básica, faz-se necessário a ampliação dos olhares e conhecimentos específicos através de uma equipe interdisciplinar que inclua o profissional fisioterapeuta, cujas ações complementarão o cuidado nessa etapa de vida.

Apesar do fisioterapeuta ter sua atuação historicamente construída na reabilitação, este profissional, segundo o Conselho Federal de Fisioterapia e Terapia Ocupacional - (COFFITO), é deveras qualificado para trabalhar em diversas áreas da saúde, atuando na promoção da saúde, prevenção de doenças e/ou agravos e reabilitação da saúde ${ }^{7}$.

$\mathrm{Na}$ atenção básica o profissional fisioterapeuta teve sua atuação consolidada e efetivada a partir da criação do Núcleo de Apoio à Saúde da Família (NASF) em 24 de Janeiro de 2008, que ampliou o escopo das ações de atenção básica com a inserção de profissionais de saúde de diferentes áreas de conhecimento, onde o fisioterapeuta atua em parceria com os profissionais da ESF${ }^{8}$. Assim, o fisioterapeuta tem se inserido no sistema de atenção básica numa perspectiva de atuação na promoção da saúde, prevenção de doenças e/ou agravos e não só no tratamento e na reabilitação, através de uma compreensão mais abrangente sobre os determinantes sociais do processo saúde doença. Sendo assim, atua de maneira articulada com os demais profissionais da equipe de saúde da família, visando acompanhar o crescimento e o desenvolvimento das crianças intervindo nas disfunções neuropsicomotoras e em outros agravos prevalentes ${ }^{9}$.

Em um estudo realizado no município de João Pessoa - PB em uma unidade de saúde da família (USF) foi possível observar que as atividades fisioterapêuticas realizadas compreenderam ações de prevenção de doenças, promoção, reabilitação e manutenção da saúde, através de intervenções individuais e coletivas. Entre as diversas ações realizadas foram citadas as interconsultas com outros profissionais da equipe em diversas linhas de cuidado incluindo a puericultura ${ }^{10}$.

Um relato de experiência vivenciada pelos residentes de fisioterapia inseridos numa USF no município de Londrina - PR apontou, dentre as funções desempenhadas por este profissional, ações para subsidiar as equipes de saúde da família no cuidado ao desenvolvimento infantil através de capacitação em reuniões de educação permanente em saúde e nas consultas de puericultura"1".

A partir da garantia do direito a saúde, é nítida a necessidade de uma assistência integral as crianças em todos os níveis de complexidade do SUS. Portanto, averiguar como a presença do fisioterapeuta na puericultura é vista pelos profissionais de uma equipe de saúde da família torna-se de grande relevância possibilitando a compreensão da importância desse profissional na visão dos demais profissionais envolvidos no cuidado destas crianças.

Diante do exposto, este estudo faz o seguinte questionamento: Qual a importância do fisioterapeuta na puericultura no olhar dos profissionais de saúde de uma unidade de saúde da família?

Observou-se uma escassez de estudos que relatam, especificamente, a atuação do fisioterapeuta na puericultura, o que também justifica a realização deste estudo contribuindo com o conhecimento científico a respeito do tema que foi abordado. Assim, diante da magnitude da temática e da escassez de material cientifico nessa área, este estudo objetivou conhecer a importância da atuação do fisioterapeuta na puericultura, na visão dos profissionais de saúde de uma unidade de saúde da família. 


\section{Metodologia}

Tratou-se de um estudo descritivo, exploratório com abordagem qualitativa dos dados. A pesquisa foi realizada em uma Unidade de Saúde da Família Integrada, no município de João Pessoa - PB, que abriga quatro equipes de saúde da família. O cenário deste estudo foi escolhido de maneira intencional, por se tratar de uma unidade que não possuía em sua equipe do NASF o fisioterapeuta no momento da realização da pesquisa. As ações fisioterapêuticas junto à comunidade nesta unidade eram realizadas através de discentes concluintes do curso de fisioterapia de uma faculdade privada e seu respectivo docente supervisor durante as atividades do estágio supervisionado. A amostra foi não probabilística, dada por conveniência e composta por 25 profissionais da referida unidade, que aceitaram voluntariamente participar da pesquisa. Todos os participantes foram abordados e convidados a colaborar com a referida pesquisa, apenas, durante o intervalo do seu expediente de trabalho ou ao seu término, não havendo, portanto, interferências ou interrupção das atividades.

Foi utilizado para a coleta dos dados uma entrevista semiestruturada contendo perguntas sobre: a) o tempo de atuação do profissional na puericultura; b) realização ou não de algum curso de aperfeiçoamento, capacitação e/ou especialização na área; c) percepção sobre o processo de trabalho entre os profissionais que atuam na puericultura; d) a importância do fisioterapeuta na puericultura e e) qual seria o papel deste profissional junto a Estratégia Saúde da Família (ESF). As entrevistas foram gravadas e transcritas por dois pesquisadores autores deste manuscrito a partir do consentimento dos participantes.

Todos os participantes assinaram o Termo de Consentimento Livre e Esclarecido e todo o percurso do estudo seguiu as normas éticas argumentas na Resolução 466/12, tendo sido aprovado pelo Comitê de Ética em Pesquisa da Faculdade de Ciências Médicas da Paraíba (protocolo n 153.204/12).

Os dados coletados foram analisados através da técnica de análise de conteúdo, temática proposta por Bardin ${ }^{12}$. As etapas descritas a seguir foram avaliadas por três experimentadores: inicialmente, foi realizada a leitura flutuante e constituição do corpus do trabalho, que foi formado por 25 entrevistas; em seguida, foi realizada a seleção das unidades de análise, considerando-se unidades de contexto os parágrafos e unidades de análise as frases; posteriormente, seguiuse o recorte, a codificação e a categorização, resultando em quatro categorias: Diagnóstico e tratamento precoce de crianças; Prevenção de doenças e outros agravos; Orientações às mães; e Avaliação do desenvolvimento das crianças.

\section{Resultados e discussão}

Dos entrevistados participantes deste estudo $52 \%$ eram agentes comunitários de saúde, $12 \%$ odontólogos, $12 \%$ assistentes de saúde bucal e $8 \%$ médicos, $8 \%$ enfermeiros e $8 \%$ técnicos de enfermagem. Vale salientar que, todos os participantes relataram realizar algum tipo de atividade relacionada à puericultura. Com relação ao tempo de atuação do profissional na puericultura, foi observado que este variou de 01 à 31 anos. Quanto a distribuição desse tempo foi possível observar que $48 \%$ dos profissionais tinham de 6 à 10 anos de atuação; 24\% de 1 à 5 anos; $12 \%$ de 11 a 15 anos; $12 \%$ de 16 a 20 anos e 4\% de 21 a 31 anos.

No entanto, quando interrogado se estes profissionais realizaram algum curso de especialização e/ou capacitação na área da temática da puericultura, $76 \%$ relataram que não realizaram. Isto se justifica por não ser obrigatória a capacitação desse profissional, para atuar especificamente na puericultura, partindo do pressuposto que cada atuante na atenção básica tem competências e habilidades suficientes para o desempenho de suas respectivas funções neste nível de atenção. Entretanto, vários autores ${ }^{13}$ assinalaram que é imprescindível, para a implementação do SUS, a existência de uma política de recursos humanos efetivos, que possibilite ações de qualificação e/ou formação permanente de seus trabalhadores. A capacitação e qualificação dos profissionais são de impar relevância para ajudar no aprendizado e aperfeiçoamento das relações sociais próprias do cotidiano dos serviços de saúde para melhorar 0 atendimento ao usuário, possibilitando consequentemente melhor desempenho nas atividades sanitárias e atenção mais qualificada para resolver os problemas que possam acontecer na população.

Quando interrogado aos entrevistados como eles percebem o processo de trabalho entre os profissionais que atuam na puericultura, foi observado que $44 \%$ percebem o processo de trabalho com interdisciplinaridade entre os profissionais da equipe; $40 \%$ apresentaram um olhar positivo ancorando suas respostas na resolutividade do atendimento, com responsabilidade e ética; $8 \%$ relataram que o processo de trabalho encontra-se em crescimento e $8 \%$ afirmaram que ainda precisa ser melhorado.

A interdisciplinaridade representa o grau mais avançado de relação entre disciplina, se considerarmos o critério de real entrosamento entre elas. Nesse caso, seriam estabelecidas relações menos verticais entre diferentes disciplinas, 
que passariam também, a compartilhar uma mesma plataforma de trabalho, operando sob conceitos em comum e esforçando-se para decodificar o seu jargão para novos colegas. 0 estabelecimento de uma relação dialógica no interior das unidades de saúde pode contribuir para a superação de relações hierarquizadas, em que os profissionais raramente conhecem as potencialidades dos outros ${ }^{14,15}$.

Quando indagados como se dá a abordagem da criança quando ela é admitida na USF estudada, 40\% afirmaram que ela é feita desde o período pré-natal; $28 \%$ relataram que as crianças são abordadas primeiramente no acolhimento que é realizado na unidade; $24 \%$ disseram que a abordagem se faz desde o nascimento; $4 \%$ responderam que a abordagem é feita através do agendamento de consultas e $4 \%$ não respondeu.

É possível observar que a assistência pode ser prestada precocemente possibilitando um atendimento mais abrangente, constituindo uma importante estratégia para a realização de mudanças significativas para o cuidado às crianças. A criança é um ser vulnerável que necessita de atenção sistemática e periódica, dessa forma a consulta da puericultura tem um papel importante no acompanhamento da criança saudável na expectativa de diminuir a incidência de enfermidades, aumentando as oportunidades para alcançar todo o potencial do crescimento e desenvolvimento. Na atenção básica a mãe tem um acompanhamento desde o seu período gestacional realizando assim o pré-natal. Logo que a criança nasce, são preconizadas sete consultas durante 0 primeiro ano de vida, duas consultas dos 12 meses aos 24 meses e uma consulta anual dos 36 aos 72 meses, consultas essas que podem ser realizadas a domicílio ou a criança é encaminhada a Unidade de Saúde da Família (USF).

Todos os entrevistados veem a puericultura no contexto do crescimento e desenvolvimento das crianças com um olhar positivo, sendo esta importante para a prevenção de doenças e para o acompanhamento do desenvolvimento e crescimento das crianças. Esta visão dos profissionais a respeito da puericultura corrobora com pesquisadores que afirmaram ${ }^{3}$ que a puericultura tem como objetivos a promoção da saúde infantil, a prevenção de doenças e a educação da criança e seus familiares, por meio de orientações antecipatórias aos riscos de deficiências ou agravos a saúde, podendo oferecer medidas preventivas mais eficazes para conhecer a criança em sua plenitude, compreendendo a criança em seu meio ambiente familiar e social.

Os dados coletados nas entrevistas submetidos à análise de conteúdo a respeito da visão dos profissionais sobre a importância da presença do fisioterapeuta na puericultura permitiram a organização das seguintes categorias: 1) Diagnóstico e tratamento precoce de crianças; 2) Prevenção de doenças e outros agravos; 3) Orientações às mães; 4) Avaliação do desenvolvimento das crianças que serão discutidas a seguir:

\section{Diagnóstico e tratamento precoce de crianças.}

Os conteúdos apreendidos demonstraram que os profissionais da equipe de saúde da família percebem a importância da atuação do fisioterapeuta junto à equipe interdisciplinar diagnosticando e tratando precocemente as crianças que apresentam algum problema motor, o que pode ser observado nas falas a seguir:

(...) Muito importante desde o nascimento para identificar doenças precocemente (...).

(...) De identificar doenças, de tratar, se for necessário, e acompanhar o desenvolvimento junto à equipe (...).

(...) Contribuir no tratamento de dificuldades de locomoção e má formação da criança proporcionando melhor qualidade de vida (...).

O profissional fisioterapeuta inserido na equipe multiprofissional da família irá contribuir na puericultura identificando precocemente alguma alteração cinético-funcional nas crianças. $O$ fisioterapeuta com conhecimentos generalistas irá aumentar a oferta de serviços aos usuários melhorando o atendimento e tratamento dessas crianças ${ }^{16}$.

O fisioterapeuta deverá atuar observando as singularidades dos indivíduos tendo como base a avaliação e o planejamento como também a execução do programa terapêutico propício para cada criança. Inicialmente o fisioterapeuta deve fazer uma avaliação, buscando identificar as limitações, as dificuldades, as alterações, as capacidades e as necessidades de cada criança. Em seguida, o tratamento deve buscar a reabilitação cinética funcional e manter as funções já existentes ou aprimorá-las ${ }^{17}$. 


\section{Prevenção de doenças e outros agravos}

Esta categoria revela em seus conteúdos que os profissionais de saúde da atenção básica veem a atuação do fisioterapeuta além da reabilitação, apontando também, a importância desse profissional em ações de prevenção de doenças e outros agravos influenciando na integralidade da atenção à saúde da criança.

\section{(...) Acompanhar o crescimento e desenvolvimento das crianças prevenindo precocemente doenças e promovendo saúde (...). \\ (...) Pra fazer prevenção de futuras doenças que podem acontecer com a criança (...). \\ (...) Observar se há alguma patologia precocemente, é melhor prevenir (...).}

Atualmente, o profissional fisioterapeuta está mudando a sua visão de atuação, deixando de ser exclusivamente reabilitador para uma atuação na atenção básica, buscando a promoção da saúde e prevenção de agravos, ou seja, no controle de risco de fatores que potencialmente podem contribuir para o desenvolvimento de doenças que comprometem a saúde do ser humano. Assim, ele pode, na USF, dentre muitas ações, participar das atividades da puericultura juntamente com os demais profissionais da equipe da unidade somando saberes e ampliando o cuidado as crianças usuárias do serviço $0^{18}$.

A necessidade da consolidação do papel do fisioterapeuta no contexto da atenção básica tem impulsionado a discussão sobre as formas de atuação deste profissional. É essencial que o profissional fisioterapeuta na Estratégia Saúde da Família (ESF) tenha uma visão para além do tratamento e da reabilitação. Este profissional deve assumir 0 encargo de agir no desenvolvimento das potencialidades do indivíduo, intervir não somente nas doenças instaladas, mas nas condições e necessidades de saúde das populações; no processo de desenvolvimento de limitações funcionais, propondo meios e recursos para evitar a instalação de situações comprometedoras da qualidade de vida.

\section{Orientaç̃̃es às mães}

A valorização e o reconhecimento dos eventos relacionados à saúde infantil e as estratégias nas decisões relativas à prevenção e ao tratamento refletem as diferentes maneiras de ter a compreensão de poder atuar sobre os problemas de saúde referente à criança. Nesta categoria foram reunidos os conteúdos que mostram que o fisioterapeuta também dá orientações às mães para um cuidado ampliado, integral e humano e que podem ser observado em alguns exemplos de falas a seguir.

\section{(...) Orientando as mães no desenvolvimento motor das crianças (...). \\ (...) Pra estimular o desenvolvimento e crescimento das crianças e orientar a mãe sobre os exercícios adequados para serem feitos com essa criança (...). \\ (...) Acompanhamento da mãe e da criança, orientações sobre a amamentação e posicionamento (...).}

(...) Na orientação da postura correta para a amamentação, tanto da mãe quanto do bebê (...).

A criança é um ser indefeso, principalmente na fase de crescimento e desenvolvimento. É nesse momento que ela precisa de cuidados para sua sobrevivência e depende da ajuda que é prestada pelo grupo social em que vive, precisando de proteção devido aos riscos contra sua vida e saúde. A mãe é muito importante na promoção da saúde da criança, ela desempenha um papel fundamental nos cuidados que são necessários para o crescimento e desenvolvimento de seu filho. Nesse sentido, é importante compreender a criança no seu contexto familiar e social, buscando escutar a família e principalmente os pais ou responsáveis pela criança, ou seja, todos que tenham uma participação constante na fase do crescimento e desenvolvimento da criança ${ }^{19}$.

O fisioterapeuta pode dar orientações às mães, quanto à importância do aleitamento materno e posicionamento delas durante a amamentação, favorecendo posturas adequadas tanto para a mãe quanto para o bebê, prevenindo problemas posturais futuros. O referido profissional também poderá orientar quanto à importância da estimulação sensório-motora da criança em fase de crescimento para aquisição do desenvolvimento neuropsicomotor normal. Assim, o fisioterapeuta 
pode utilizar das diversas técnicas e recursos próprios da fisioterapia para alcançar seus objetivos junto às crianças e as mães e esse atendimento pode ir além da unidade ampliando assim os atendimentos as crianças e orientações às mães no seu ambiente domiciliar ou até mesmos nas creches ${ }^{20}$.

\section{Avaliação do desenvolvimento neuropsicomotor das crianças}

Os conteúdos desta categoria revelam o reconhecimento dos profissionais entrevistados a cerca das habilidades e competências que o fisioterapeuta possui para a avaliação e acompanhamento do desenvolvimento neuropsicomotor das crianças nos diversos níveis de atenção a saúde, incluindo a atenção básica, como pode ser observado em algumas falas a seguir.

(...) Identificar com maior percepção reflexos necessários para o crescimento e desenvolvimento da criança, atuando junto à equipe que participa da puericultura (...).

(...) Observar o desenvolvimento motor da criança em relação ao seu crescimento (...).

(...) Os profissionais fisioterapeutas, no processo de desenvolvimento motor são de fundamental importância (...).

O desenvolvimento humano é um processo de crescimento e mudanças, seja do comportamento, cognitivo e emocional ao longo da vida. O crescimento e desenvolvimento da criança têm várias fases e devido a isso, surgem características especificas. Cada criança é um indivíduo único e vivencia estas fases de diferentes maneiras de acordo com as suas experiências desde a vida intrauterina. A criança precisa de rotinas de planejamento, estrutura e organização para que ela venha ter um melhor desenvolvimento ${ }^{5}$.

Quanto ao acompanhamento do desenvolvimento neuropsicomotor (DNPM) da criança, o fisioterapeuta realiza avaliações fisioterapêuticas individuais das crianças, identifica precocemente atraso no DNPM e encaminha o mais rápido possível para um serviço de referência para o tratamento fisioterapêutico adequado, prevenindo complicações.

\section{Considerações Finais}

Os resultados obtidos com este estudo inferem que a atuação do fisioterapeuta é importante e imprescindível para a efetivação do cuidado multidimensional na puericultura e que os profissionais de saúde reconhecem a presença do fisioterapeuta junto à equipe, atuando na puericultura, de grande valor, principalmente para a integralidade do cuidado a saúde das crianças.

Partindo do princípio de que uma das prioridades da ESF é o desenvolvimento integral da criança e as ações realizadas na puericultura, pela equipe de saúde, visam, principalmente, a prevenção de doenças e outros agravos nos primeiros anos de vida, torna-se imprescindível à atuação do fisioterapeuta neste nível de atenção, tendo em vista que sua formação profissional, pode influenciar positivamente na integralidade da atenção à saúde da criança, agregando elementos necessários à intervenções e ao cuidado.

Portanto, a missão da fisioterapia nesse contexto é primordial diante da consolidação do seu papel na atenção básica e da nova realidade de saúde que se apresenta, através dos recursos terapêuticos físicos, na extinção de estados patológicos do indivíduo, na educação e promoção em saúde e na prevenção de doenças e outros agravos.

\section{Referências Bibliográficas}

1. Ministério da Saúde (BR). Secretaria Nacional de Assistência á Saúde. ABC do SUS - comunicação visual. Brasília: Secretaria Nacional de Assistência à Saúde, 1991.

2. Ministério da Saúde (BR). Estatuto da Criança e do Adolescente. $3^{a}$ ed. Brasília: Ministério da Saúde, 2008.

3. Ciampo LAD, Ricco RG, Daneluzzi JC, Ciampo IRLD, Ferraz IS, Almeida CANO. Programa de Saúde da Família e a Puericultura. Ciênc. saúde coletiva. 2006; 11(3):739-743.

4. Campos RMC, Ribeiro CA, Silva CV, Campos E, Saparolli L. Consulta de enfermagem em puericultura: a vivência do enfermeiro na Estratégia de Saúde da Família. Rev. Esc. Enferm. USP. 2011;45(3):566-574. 
5. Vasconcelos VM, Frota MA, Martins MC, Machado MMT. Puericultura em Enfermagem e Educação em Saúde: percepção de mães na estratégia saúde da família. Esc. Anna Nery Rev. Enferm. 2012;16(2):326-331.

6. Piccini RX, Facchini LA, Tomasi E, Thumé E, Silveira DS, Siqueira FV, Rodrigues MA, Paniz VV, Teixeira VA. Efetividade da atenção pré-natal e de puericultura em unidades básicas de saúde do Sul e do Nordeste do Brasil. Rev. Bras. Saude Mater. Infant. 2007;7(1):75-82.

7. Cogo LA, Freitas CS, Ribeiro JS, Vogt MSL, Miolo SB. Percepção dos agentes comunitários de saúde sobre a fisioterapia na atenção primária. Saúde (Santa Maria). 2013;39(1):101111.

8. Ministério da Saúde (BR). Secretaria de Atenção à Saúde. Departamento de Atenção Básica. Núcleo de Apoio à Saúde da Família. Brasília: Ministério da Saúde, 2014.

9. Gama KCSD. Inserção do Fisioterapeuta no Programa de Saúde da Família: Uma Proposta Ética e Cidadã. C\&DRevista Eletrônica da Fainor. 2010;3(1):12-29.

10. Sousa ARB, Ribeiro KSQS. A Rede Assistencial em Fisioterapia no Município de João Pessoa: uma análise a partir das demandas da atenção básica. Rev. Bras. de Ciências da Saúde. 2011;15(3): 357-368.

11. Yonamine CY, Trelha CS. $O$ modo de fazer saúde: a fisioterapia na residência multiprofissional em saúde da família em uma unidade básica. Rev. Espaço para a Saúde, Londrina. 2009;11(1):17-27.

12. Bardin L. Análise de conteúdo. Lisboa: Edições 70, 2004.

13. Cotta RMM, Schott M, Azeredo CM, Franceschini SCC, Priore SE, Dias G. Organização do trabalho e perfil dos profissionais do Programa Saúde da Família: um desafio na reestruturação da atenção básica em saúde. Epidemiol. serv. saúde. 2006;15(3):7-18. 14. Iribary IN. Aproximações sobre a transdisciplinalidade: Algumas linhas históricas, fundamentos e princípios aplicados ao trabalho de equipe. Psicol. reflex. crit. 2003;16(3):483-490.

15. Peduzzi M. Equipe Multiprofissional de saúde: conceito e tipologia. Rev. saúde pública. 2001;35(1):103-109.

16. Rocha VM, Caldas MAJ, Araújo FRO, Ragasson CAP, Santos MLM, Batiston AP. As diretrizes curriculares e as mudanças na formação de profissionais fisioterapeutas (ABENFISIO). Fisioter. Bras. 2010;11(5):4-8.

17. Fujisawa DS, Manzini EJ. Formação Acadêmica do Fisioterapeuta: a utilização das atividades lúdicas nos atendimentos de crianças. Rev. bras. educ. espec. 2006;12(1):65-84.

18. Bispo PJ Jr. Fisioterapia e saúde coletiva: desafios e novas responsabilidades profissionais. Ciênc. saúde coletiva. 2010;15 Supl1:1627-1636.

19. Ximenes Neto GRF, Queiroz CA, Rocha J, Cunha ICKO. Por que não levo meu filho para consulta da puericultura. Rev. Sociedade Bras. de Enfermeiros Pediatras. 2010;10(2):51-59.

20. Abreu ARBR, Apa MFM, VAL LF. Fisioterapia: a importância na estratégia saúde da família. In: IV Simpósio Internacional de Ciências Integradas da UNAERP Campus Guarujá. 2007. Disponível em: http:// http://www.unaerp.br/ index.php/documentos/1036-fisioterapia-a-importancia-na-estrategia/file. Acesso em: 28/06/2012.

\section{Olivia Galvão Lucena Ferreira}

Endereço para correspondência - Praça Dr. Aquiles Leal, nº 49, Bairro: Jaguaribe, CEP: 58015-450 João Pessoa, PB, Brasil.

E-mail: oliviaglf@hotmail.com

Lattes: http://lattes.cnpq.br/2504787116305308

Turrania Talita Soares de Castro - turrania.castro@hotmail.com

Simone Felipe Santiago - simonefisio2012@hotmail.com

Sandra Fernandes Pereira Meló - sandrafermelo@hotmail.com

Egmar Longo Araújo de Melo - egmarlongo@hotmail.com

Verbena Santos Araújo - verbena.bio.enf@hotmail.com

Enviado em 16 de fevereiro de 2014. Aceito em 29 de junho de 2015. 
